\title{
Diverse protein kinase interactions identified by protein microarrays reveal novel connections between cellular processes
}

\author{
Joseph Fasolo, ${ }^{1,2}$ Andrea Sboner, ${ }^{3,4}$ Mark G.F. Sun, ${ }^{5,6}$ Haiyuan Yu, ${ }^{7,8}$ Rui Chen, ${ }^{1}$ Donald Sharon, ${ }^{1,2}$ \\ Philip M. Kim, ${ }^{5,6,9,10}$ Mark Gerstein, ${ }^{3,4,11}$ and Michael Snyder ${ }^{1,2,4,12}$ \\ ${ }^{1}$ Department of Genetics, Stanford University School of Medicine, Stanford, California 94305, USA; ${ }^{2}$ Department of Molecular, \\ Cellular and Developmental Biology, Yale University, New Haven, Connecticut 06520, USA; ${ }^{3}$ Program in Computational \\ Biology and Bioinformatics, Yale University, New Haven, Connecticut 06520, USA; ${ }^{4}$ Department of Molecular Biophysics and \\ Biochemistry, Yale University, New Haven, Connecticut 06520, USA; ${ }^{5}$ Donnelly Centre for Cellular and Biomolecular Research, \\ University of Toronto, Toronto, Ontario M5S3E1, Canada; ${ }^{6}$ Department of Computer Science, University of Toronto, Toronto, \\ Ontario M5S3E1, Canada; ${ }^{7}$ Department of Biological Statistics and Computational Biology, Cornell University, Ithaca, New \\ York 14853, USA; ${ }^{8}$ Weill Institute for Cell and Molecular Biology, Cornell University, Ithaca, New York 14853, USA; ${ }^{9}$ Banting \\ and Best Department of Medical Research, University of Toronto, Toronto, Ontario M5S3E1, Canada; ${ }^{10}$ Department of \\ Molecular Genetics, University of Toronto, Toronto, Ontario M5S3E1, Canada; ${ }^{11}$ Department of Computer Science, Yale \\ University, New Haven, Connecticut 06520
}

Protein kinases are key regulators of cellular processes. In spite of considerable effort, a full understanding of the pathways they participate in remains elusive. We globally investigated the proteins that interact with the majority of yeast protein kinases using protein microarrays. Eighty-five kinases were purified and used to probe yeast proteome microarrays. One-thousand-twenty-three interactions were identified, and the vast majority were novel. Coimmunoprecipitation experiments indicate that many of these interactions occurred in vivo. Many novel links of kinases to previously distinct cellular pathways were discovered. For example, the well-studied Kss1 filamentous pathway was found to bind components of diverse cellular pathways, such as those of the stress response pathway and the Ccr4-Not transcriptional/translational regulatory complex; genetic tests revealed that these different components operate in the filamentation pathway in vivo. Overall, our results indicate that kinases operate in a highly interconnected network that coordinates many activities of the proteome. Our results further demonstrate that protein microarrays uncover a diverse set of interactions not observed previously.

[Keywords: Kss1 MAPK; interaction network; protein kinase; protein microarrays; proteomics; yeast]

Supplemental material is available for this article.

Received October 3, 2010; revised version accepted February 14, 2011.

Complex cellular and developmental processes are executed through the intricate control of numerous cellular and molecular pathways. Understanding the regulatory mechanisms that coordinate metabolic and adaptive processes requires dissection of the molecular networks that mediate gene expression, protein-protein interactions, and post-translational modifications. Protein kinases are key regulators of molecular pathways, and it is estimated that $\sim 50 \%$ of all cellular proteins are phosphorylated in vivo (Kornev and Taylor 2010). A comprehensive understanding of the proteins that interact with protein

${ }^{12}$ Corresponding author.

E-MAIL mpsnyder@stanford.edu; FAX (650) 331-7391.

Article is online at http://www.genesdev.org/cgi/doi/10.1101/gad.1998811. Freely available online through the Genes \& Development Open Access option. kinases is crucial for determining the pathways in which the different kinases operate and how they are regulated.

Previous system-wide analysis of protein-protein interactions using affinity capture techniques coupled with mass spectrometry (AC/MS) (Gavin et al. 2002; Ho et al. 2002; Krogan et al. 2006; Breitkreutz et al. 2010) and twohybrid technologies (Uetz et al. 2000) have identified numerous interactions with yeast protein kinases and other signaling components. However, in general, largescale studies using different approaches, such as AC/MS and two-hybrid analyses, or even using the same general approach, have revealed little overlap between data sets (e.g., Yu et al. 2008). Thus, the catalog of interactions is far from complete, and considerably more effort is required to obtain a comprehensive and accurate assignment of kinases to cellular pathways. 
In our study, we investigated the proteins that interact with yeast protein kinases using protein microarrays. Protein microarrays offer the advantage that the arrays contain nearly all yeast proteins, and they can be assayed simultaneously for biochemical interactions in vitro (Zhu et al. 2001). In contrast, many other methods, such as $\mathrm{AC} / \mathrm{MS}$, primarily detect interactions between the most abundant proteins, and may also be biased toward identification of those components in stable complexes. Therefore, it is likely that new interactions could be discovered using protein microarrays. However, thus far, only limited studies to globally analyze proteinprotein interactions have been performed using this approach (Zhu et al. 2001; Popescu et al. 2007).

In this study, we employed a large-scale approach using protein microarrays to identify yeast kinase-protein interactions. We discovered and validated many novel interactions, revealing an entirely new set of functional links between kinases and cellular pathways. Such information was shown to provide new insights into even well-studied biological processes such as the filamentation signaling pathway.

\section{Results}

\section{In vitro protein kinase-binding profiles}

To identify proteins that interact with protein kinases using proteome microarrays, 109 of the $\sim 125$ yeast protein kinases (Zhu et al. 2001; Breitkreutz et al. 2010) were tagged with a C-terminal V5 epitope. Eighty-five of these were successfully purified in sufficient quantities and purity to probe a yeast proteome microarray containing $\sim 4200$ unique yeast proteins (Fig. 1; Table 1; see the Materials and Methods). Each kinase was assayed using two protein microarrays, along with a negative control (see the Materials and Methods), and the overlapping interactions for each duplicate experiment were analyzed using modified ProCAT software (Zhu et al. 2006). Using stringent criteria (described in the Materials and Methods), we identified 1023 interactions involving 425 different proteins (Supplemental Table S1). The number of interacting proteins for each kinase ranged from one to 47 (median $=9)$. The majority of targets bound a single kinase, although 198 proteins bound more than one kinase (Supplemental Fig. S1).

\section{Coimmunoprecipitation (co-IP) validation of targets in vivo}

To evaluate whether the proteins bound on the array also interact with the protein kinases in vivo, we tested interactions for nine kinases from distinct subfamilies using co-IP experiments. Forty-five strains were constructed that contain a protein kinase, tagged at its $\mathrm{C}$ terminus with $13 \mathrm{XMyc}$, and the interacting protein identified from the protein array, tagged at its $\mathrm{C}$ terminus with a TAP tag (Rigaut et al. 1999). These included 20 proteins that interacted with 10 or more kinases (two of these were tested for interactions with multiple kinases). The 45 strains contained proteins whose kinase interac-

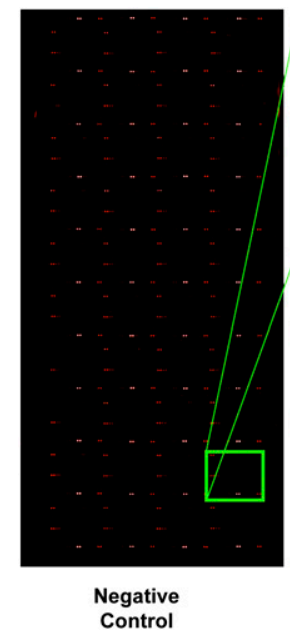

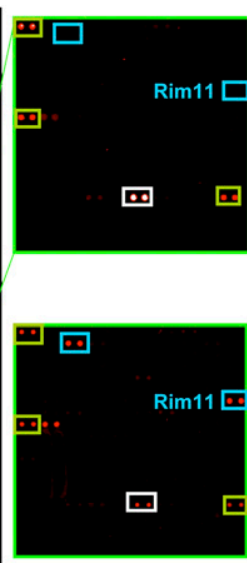

Control

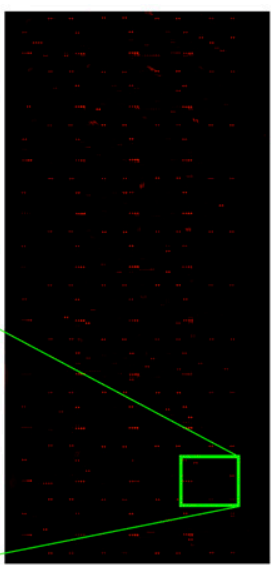

Ymr291w-V5
Figure 1. Protein microarrays probed with negative control and Ymr291w-V5 preparations. Yeast functional protein microarrays containing $\sim 4200$ unique full-length GST fusion yeast proteins spotted in duplicate were probed with kinase-V5 probes. Two arrays were probed for each kinase, and duplicate signals present on the kinase-probed array relative to the control arrays were identified (see the Materials and Methods). Inset panels compare the same region on both arrays. Anti-mouse antibodies conjugated with fluorescent dye were spotted on the arrays to provide positioning coordinates (green boxes). Control fusion proteins with V5-epitope tag are also included (white boxes). We used purified lysates prepared from yeast transformed with empty pYES-DEST52 vector as our negative control. Protein-protein interactions are indicated by the blue boxes. The interaction between Rim11 and Ymr291w was validated in vivo by co-IP/immunoblot and is not present in negative control arrays.

tions were detected at a wide range of intensities on the protein microarrays. A strain with tagged Crzl, which was known previously to interact with Hrr25 (Kafadar et al. 2003), was also included as a positive control. In each case, the proteins were expressed from native loci to assess the binding interactions at their endogenous levels. Protein complexes were isolated from crude yeast cell extracts using IgG-coupled magnetic beads to capture the TAP-tagged target protein, and the immunoprecipitates were examined for the presence of 13XMyc protein kinases by immunoblot analysis. Negative controls included the untagged kinase and untagged interacting protein strains. A total of 32 novel protein-protein interactions identified in the protein microarray screen were confirmed by co-IP (Fig. 2), as was the positive control. The negative control strains did not coimmunoprecipitate. Of the 13 that failed (Supplemental Table S2), 10 involved the proteins that interacted with $>10$ kinases and are likely to be nonspecific interactions on the original array; the remaining three proteins interacted with $<10$ kinases. For the three proteins that appear to be specific (i.e., interacted with $<10$ proteins on the array) but did not validate using co-IP, their lack of interaction cannot be readily interpreted; they might interact under other growth/environmental conditions. In summary, 20 
Table 1. Kinases tested in this study sorted by major kinase groups

\begin{tabular}{ll}
\hline Group & \multicolumn{1}{c}{ Kinases tested } \\
\hline AGC & Cbk1, Dbf2, Dbf20, Kin82, Pkh2, Phk3, Sch9, Tpk1, Tpk2, Tpk3, Ybr028c, Ypk1 \\
Atypical PK & Rio1 \\
CAMK & Cmk1, Cmk2, Elm1, Frk1, Gin4, Hs11, Kcc4, Kin1, Kin2, Mek1, Rck1, Rck2, Snf1, Ymr291w, Tos3, Ypl150w \\
CAMK/EMK & Prr1 \\
CK1 & Hrr25, Yck2, Yck3 \\
CMGC & Cak1, Cdc28, Ctk1, Ctk2^, Fus3, Kin28, Kss1, Mck1, Mrk1, Rim11, Sky1, Slt2, Smk1, Ssn3, Yak1, Ygk3 \\
Other & Akl1, Ark1, Cdc7, Chk1, Gcn2, Hal5, Ip11, Kkq8, Ksp1, Mps1, Npr1, Prr2, Prs5, Psk2, Ptk2, Rtk1, Sat4, Scy1, \\
& Sks1, Swe1, Vhs1 \\
STE & Bck1, Cdc15, Cla4, Kin3, Mkk1, Pbs2, Skm1, Sps1, Ssk2, Ste7, Ste11, Ste20
\end{tabular}

Eighty-five kinases were used to screen for protein-protein interactions on the protein arrays. The kinases were sorted according to their taxonomical grouping: AGC (cyclic nucleotide-dependent family [PKA and PKG] and the protein kinase C), Atypical PK, CAMK (calcium/calmodulin), CAMK/EMK (CAMK/ELKL motif kinase), CK1 (casein kinases), CDKs (cyclin-dependent kinases), MAP kinases (mitogen-activated protein kinases), CMGC (glycogen synthase kinases [GSK] and CDK-like kinases), other (kinases that do not have sequence similarity with any other kinase), STE (sterile homologs), and Yeast PK (kinases unique to yeast). Ctk2 ${ }^{\star}$ is the $\beta$ subunit of C-terminal domain kinase 1 (CTDK1) kinase.

out of $23(83 \%)$ specific interaction sets (those that bound $<10$ kinases) were validated, whereas only half (10 out of 20 ) of the less specific interaction sets (those that bound $>10$ kinases) were validated. Given the success rate for validation of targets bound more specifically to kinases tested in our screen, the data were filtered to include only those interactions occurring between $<10$ kinases on the protein microarray; our data suggest that the majority of these interactions occur in vivo.

The interactions identified by protein microarrays are distinct from those found by other methods

Our study is the largest interaction screen performed to date using protein microarrays. Several other global studies to identify proteins that interact with protein kinases have been performed; notably AC/MS studies and two-hybrid studies. Surprisingly, in spite of the extensive in vivo validation we performed, only a small fraction of the interacting proteins found using the protein microarrays overlap with those of the other studies. We found that only two of 1023 interactions overlapped with earlier genome-wide co-IP studies, and nine overlapped with a very recent and thorough kinase AC/MS study (Fig. 3A; Breitkreutz et al. 2010). Similarly, only three of 1023 of our interactions overlap with a recent two-hybrid study (Yu et al. 2008). These results indicate that the interacting partners identified using protein microarrays are distinct from those found using other methods.

We suspect that the minimal overlap of our results with those identified by other methods is likely due to the fact that our method identifies binary interactions, whereas AC/MS identifies entire complexes. To explore this possibility further, we analyzed the frequency with which kinase-interacting proteins had interactions among themselves, as reported in BioGRID (Stark et al. 2006). We found that, of the 425 interacting proteins, 12 interacted with other interacting proteins of the same kinase from BioGRID (an example of the most connected one is shown in Supplemental Fig. S2A). In contrast, the affinity capture data set of Breitkreutz et al. (2010) had 1844 partnerpartner interactions in 887 distinct kinase-interacting proteins (an example is shown in Supplemental Fig. S2B). Thus, these results suggest that the AC/MS experiments identify interacting sets of proteins that are part of the same complex, whereas the protein array identifies binary interacting partners.

We analyzed our interaction sets to see whether they might be targets of phosphorylation of the interacting kinase using two methods. First, we compared the interaction data with the set of kinase substrates identified using similar protein microarrays (Ptacek et al. 2005). We found only 34 protein targets in common between the interaction and phosphorylation data sets, indicating that these are mostly distinct sets of proteins; in addition, the phosphorylation data set generated from protein microarrays is likely incomplete. To further explore whether the kinase partners are potentially phosphorylated by their interacting kinase, we computationally examined each interacting partner for the presence of high-scoring phosphorylation motifs using the Mok et al. (2010) data set (see the Materials and Methods). We found that 25\% of the interacting proteins had high-confidence phosphorylation motifs for their partner kinase; this figure is significantly enriched over random $\left(P\right.$-value $\left.<10^{-20}\right)$, but is lower than that observed by Breitkreutz et al. (2010) (50\%). It nonetheless indicates that many interacting partners are likely to be kinase substrates. We do not expect $100 \%$ overlap, since interacting proteins identified by protein microarrays may contain divergent phosphorylation sites or contain domains that bind the kinase and are not phosphorylated. Markov clustering (van Dongen 2000) was performed on the BioGRID protein-protein interaction set (Stark et al. 2006) to determine cluster membership for kinase interactions with high-confidence phosphorylation motifs in both the Breitkreutz et al. (2010) and the protein microarray data sets (Supplemental Table S3). In both cases, a majority of the kinases' interacting partners belong to the same functional cluster, indicating that kinase interactions with high-confidence phosphorylation motifs are highly likely to function in the same cellular process. 
A.

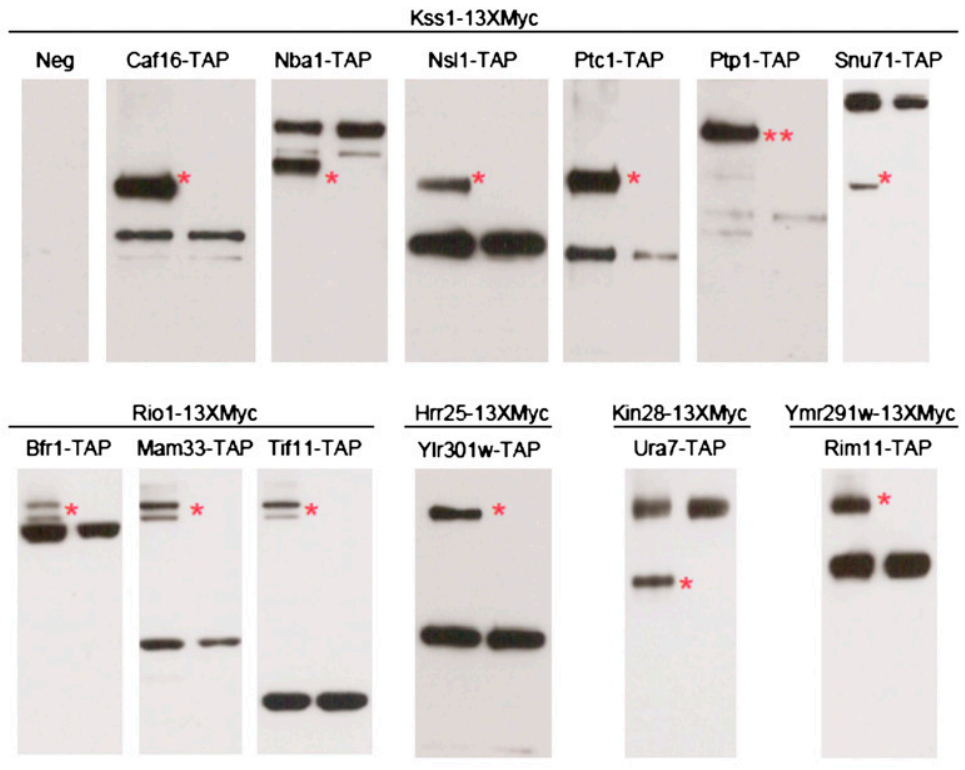

B.

\begin{tabular}{|l|l|}
\hline 13XMyc-Kinase & TAP-Tagged Target \\
\hline Hrr25 & Crz1, Hri1 (Ylr301w), Pcl10, Tmt1 \\
\hline Ipl1 & Arp8, Rck2, Tif11, Ykl091c \\
\hline Kin28 & Sct1, Ura7 \\
\hline Kss1 & $\begin{array}{l}\text { Caf16, Cta1, Nba1, Nsl1, Pof1 (Ycl047c), Ptc1, Ptp1, } \\
\text { Snu71 }\end{array}$ \\
\hline Rck2 & Apn1 \\
\hline Rio1 & Bfr1, Mam33, Nop53, Pah1, Rkm4, Tif11 \\
\hline Snf1 & Swm2, Ybr096w \\
\hline Ymr291w & Rim11, Ytp32 \\
\hline Vhs1 & Ack1, Bna1, Sip2, Ubp7 \\
\hline
\end{tabular}

Figure 2. Co-IP/immunoblot validation of kinasebound target proteins confirms interactions observed on the proteome arrays in vivo. $(A)$ Kinase$13 \mathrm{XMyc} /$ target protein-TAP strains are used to test protein microarray interactions in vivo using co-IPcoupled immunoblot analysis. The membranes were probed with 9E10 anti-Myc antibody followed by secondary IgG-HRP. Targets that interact with Kss1, Rio1, Hrr25, Kin28, and Ymr291w on the protein microarrays were coimmunoprecipitated with their cognate kinase (left lane) and and compared with coIP of the target alone (right lane). The experimental co-IPs were also compared with a negative control (Neg) co-IP lane containing a sample prepared from a strain harboring the tagged kinase alone (Kss1, first panel; data not shown for Rio1, Hrr25, Kin28, and Ymr291w). A red asterisk $\left({ }^{\star}\right)$ indicates the presence of kinase-13XMyc in the co-IP strain confirmed using an anti-myc immunoblot. The Kss1/Ptp1 co-IP resulted in a shift of Kss1 gel migration, as indicated by two asterisks $\left(^{\star \star}\right)$. The Ptp1 band is present and the Kss1-13Xmyc band is absent when the membrane is probed with secondary antibody-HRP alone (data not shown), thereby confirming the identity of the Ptp1 shifted protein. (B) Summary of 33 novel protein interactions identified on the protein microarray validated by co-IP/immunoblot (Hrr25 and Crz1 served as a positive control). The protein designated Ylr301w has no known cellular role in vivo and binds to Hrr25. We propose naming Ylr301w Hri1 (for Hrr25-interacting protein).

\section{Distinct and overlapping sets of interactions revealed by different members of kinase families}

Yeast has a number of protein kinases that are closely related by sequence and/or function to one another. An example is the Nim1-like kinases Gin4, Hsl1, and Kcc4, which are functionally redundant in the cell cycle checkpoint that responds to defects in the peripheral cytoskeleton (Barral et al. 1999). We found that the Nim1-like kinases shared some targets, but the majority of targets are kinase-specific (Fig. 4A). One interesting example is Ygr017w, which was bound by Hsl1 and Gin4. Swe1, which is in the same pathway as Hsll and Gin4, phosphorylates Ygr017w (Ptacek et al. 2005). Nonetheless, the vast majority of Gin4, Hsl1, and Kec4 have distinct targets, which is consistent with the unique phenotypes of the different kinases in pseudohyphal growth and cell cycle control (La Valle and Wittenberg 2001).

Analyses of the targets from very different kinase families revealed an unexpected result: Different types of kinases can often bind the same targets. An example is Akl1, in which 10 of 24 targets overlap with the 29 targets of Snf1 $(P<1.15 \mathrm{E}-16)$ (Fig. 4B). Akl1 is involved in cytoskeletal function and Snfl is involved in carbon metabolism; these two processes are themselves linked (see Costigan and Snyder 1994). Another example is Ipl1, for which nine targets bind one or more members of the Nim1 kinase family (Hsl1, Gin4, and Kcc4; $P<1.20 \mathrm{E}-12$; two of these nine were validated to interact with Ipl1) (Fig. 4A). Thus, distinct types of kinases often share common targets, suggesting that they may coordinate the same types of processes, either cooperatively or antagonistically. To our knowledge, this is the first report of different families of kinases sharing significant overlap in their binding of proteins involved in multiple cellular processes, which are expected to be coordinated.

\section{Novel interactions with Kss1 suggest the filamentation pathway is associated with a diverse set of biological processes}

Many interesting interactions were observed, as shown in Figures 5 and Supplemental Figure S3. One example is the Riol protein kinase involved in rRNA processing (Vanrobays et al. 2001). We found that Riol interacts with proteins involved in rRNA processing and translation; several of these interactions were validated (Supplemental Fig. S3). Examples include Tif11 (a translation initiation factor), $\mathrm{Rkm} 4$ (a ribosomal lysine methyltransferase), Nop53 (a protein involved with the biogenesis of the 60S subunit of the ribosome), and Bfrl (a component of mRNP complexes). Thus, Riol is likely to mediate its 
A.

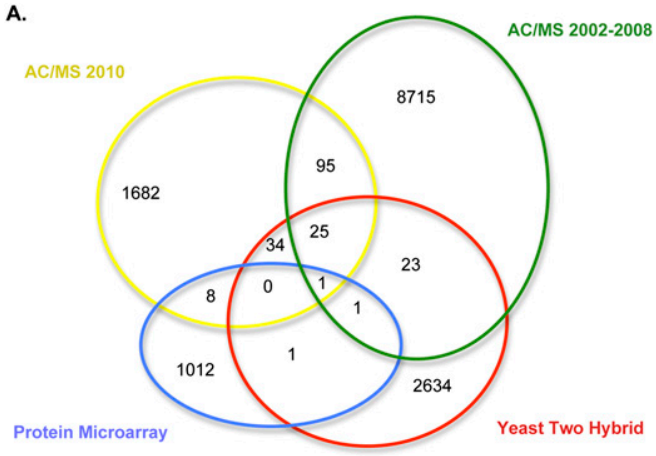

B.

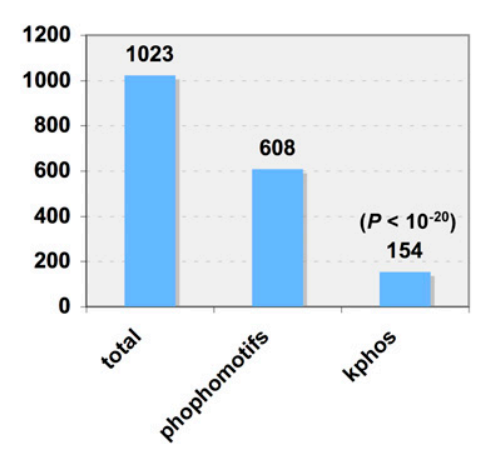

Figure 3. Comparison of interactions from multiple highthroughput protein-protein interaction studies. $(A)$ Interactions detected on the protein array (blue) were compared with the 2002-2008 AC/MS (green), yeast two-hybrid (red), and the most recent $2010 \mathrm{AC} / \mathrm{MS}$ (yellow) data sets. Note: This diagram is not drawn to scale and only indicates the overlap between data sets. (B) Plot of targets containing phosphomotifs. There were 1023 interactions (total), 608 of which contain phosphomotifs identified by Mok et al. (2010) (no position weight matrics [PWM] available for 415 proteins) (data not shown). There are 154 proteins that contain the corresponding phosphomotifs for the kinases bound to them (kphos). There are 154 out of 608 (25\%) possible kphos interactions $(25 \%)$, which is significantly enriched over random $\left(P\right.$-value $\left.<10^{-20}\right)$.

functions, at least in part, through these components, and may help coordinate rRNA processing and translation.

One of the most interesting sets of interactions involved the Kss1 kinase, which controls filamentous growth and the pheromone response and has been highly studied (Cook et al. 1997; Erdman and Snyder 2001). Kss1 interacted with a diverse set of proteins on the proteome array, many of which were validated by co-IP/immunoblot analysis. These Kss1 interactions include two phosphatases (Ptc1 [which is involved in the Hog1-osmosensing pathway] and Ptp1 [a phosphotyrosine-specific phosphatase with broad substrate specificity]), a protein component of the CCR4-NOT transcriptional regulatory complex (Caf16), a component of the MIND kinetochore complex (Nsl1, which has been implicated in cell cycle progression), and two proteins with unknown functions
(Ycl047c and Nba1). As demonstrated below, Ycl047c promotes filamentation, and we renamed it Pof1 (for promoter of filamentation). The complexes identified in our screen between Kss1 and this group of targets had not been identified previously using AC/MS or two-hybrid approaches, nor have they been directly implicated in filamentation signaling. However, these proteins are highly connected among themselves, and they also interact with other proteins implicated in filamentation, such as the phosphatase Glc7 (Fig. 5). Our results indicate that Kss1 operates with a diverse suite of components, such as those involved in transcriptional regulation, the Hogl stress response, and the kinetochore/cell cycle progression.

We did not identify many known binding partners of Kss1 because they either were not present on the array (Ste7, Ste11, Ste12, and Tec1) or were present at very low abundance (Dig1 and Dig2, which were $\sim 175$-fold and $\sim 11$-fold less, respectively, than other binding proteins on the array). Nonetheless, our ability to detect and validate many new Kssl-interacting partners indicates a much broader involvement in other pathways (see the Discussion).

Kss1-interacting proteins operate in the filamentation pathway

The results presented above suggest a highly interactive network between Kss1 and a variety of different cellular components. We examined whether any of the newly identified interacting partners operate in the same pathways as KSS1 using genetic tests. We specifically tested the validated targets from the Kss1 data sets for their ability to operate in the haploid invasive growth pathway, which requires Kss1 (Cook et al. 1997; Erdman and Snyder 2001). When haploid yeast cells are deprived of a fermentable carbon source, the cells adopt an elongated cellular morphology and invade agar (Cook et al. 1996; Lorenz et al. 2000). We tested whether the protein factors that interact with Kss1 in our screen affected filamentation and/or suppressed the filamentation defects of a kss1 deletion strain. PTC1, CAF16, POF1, NSL1, and PTP1 (along with vector, SNU71, CTA1, and RIM9, which proved to be negative controls) were expressed from the GAL1 promoter on a high-copy plasmid and transformed into wild-type $(\Sigma 1278 \mathrm{~b})$ and kss1 $1 \mathrm{~h}$ haploid yeast. Expression was induced by growth on galactose, and the ability of the cells to invade agar was examined (Fig. 6A). Wildtype cells invaded agar, whereas kss1s cells did not. Overexpression of PTC1, CAF16, POF1, and NSL1 each partially suppressed the phenotype of kss1s cells, suggesting that they function independently of or downstream from Kss1. Overexpression of SNU71, CTA1, and RIM9 in the kss1s background did not facilitate invasive growth (Fig. 6; data not shown). Furthermore, microscopic examination of kss1 $1 \Delta$ yeast overexpressing CAF16, NBA1, NSL1, and PTC1 revealed the elongated pseudohyphal cellular morphology, consistent with haploid invasive growth (Fig. 6B); negative control strains did not. The fact that PTC1, a negative regulator of the HOG1-osmosensing pathway, also operates downstream from KSS1 indicates 
A. Hsl1

Gin4

Kcc4

Ipl1

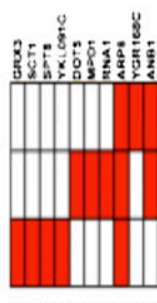

$\frac{1}{3}$
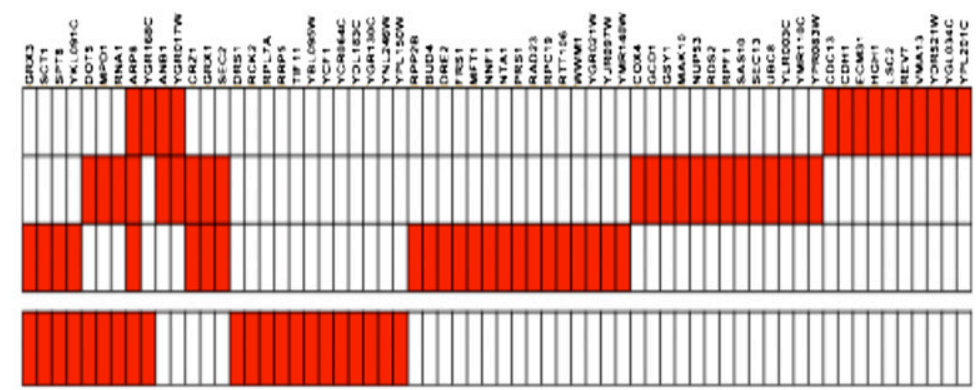

(1)
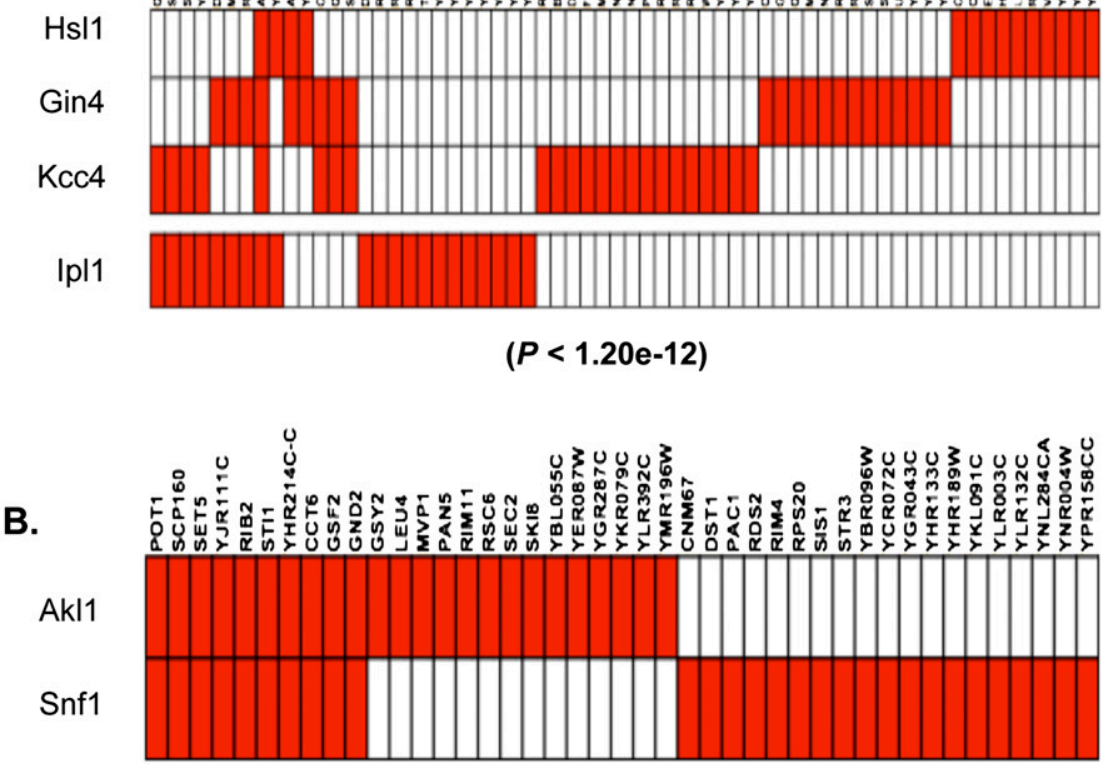

Figure 4. Comparison of overlapping interactions between similar and distinct families of kinases. Targets corresponding to each kinase are highlighted in red to allow for comparison of binding profiles. (A) Although the Nim1-like kinases (Gin4, Hsl1, and Kcc4) all function redundantly in the septin/cell cycle checkpoint, most of the proteins detected to interact with them in the screen demonstrate distinctive binding specificity. The Ipll aurora kinase demonstrates a significant level of complementarity $(P<1.20 \mathrm{E}-12)$ with nine targets bound across all three of the Nim1-like kinases. (B) A comparison of Ak11 and Snf1 reveals a significant overlap $(P<1.15 \mathrm{E}-16)$ of 10 out of 29 targets.

$(P<1.15 \mathrm{e}-16)$

a further genetic link between these pathways (see the Discussion).

Interestingly, overexpression of PTP1 in wild-type cells suppressed haploid invasion of wild-type cells, suggesting that it is a negative regulator of filamentation (Fig. 6A). PTP1 overexpression also suppressed pseudohyphae in the wild-type background (Fig. 6B). Overexpression of genes in wild-type cells for the other target proteins (PTC1, CAF16, YCL047c, NSL1, SNU71, CTA1, NBA1, and RIM9) had minimal to no effect on invasion or morphology. Overall, our results suggest that Kss1 operates directly or indirectly through a diverse array of cellular activities, which include elements of the kinetochore and Ccr4-Not-regulated transcriptional/translational control. We also identified interactions with several important protein phosphatases that have a broad array of substrates in vivo (Fig. 6).

\section{Global interaction network and network modules}

The interactions identified in our screen were used to generate a first-generation protein microarray kinase physical interaction network (Fig. 7). Raw kinase interactions were processed through a two-step learning algorithm to produce a final high-quality kinase interaction set (see the Materials and Methods, data analysis). As noted above, almost half of the targets (198 out of 425) interact with multiple kinases, and further analysis of kinase-kinase interactions and those with common interacting proteins revealed that, overall, the kinases are highly connected with one another. In fact, there are more interactions with multiple kinases than those that interacted uniquely with a single kinase, consistent with the recent study by Breitkreutz et al. (2010). However, our data are mostly unique relative to those of Breitkreutz et al. (2010), suggesting that the density of interactions is much higher than reported previously.

We subsequently determined that the protein microarray kinase interaction data set was enriched for network modules (Supplemental Table S4). First, we used the Mok et al. (2010) data set to identify interactions due to kinase phosphorylation (kphos) or binary protein-protein interactions (kbin). Second, we integrated our kinase interaction data (Fig. 7A) with transcription factor binding and protein interactions between kinase-interacting partners (Fig. 7B) to generate a "metanetwork" of 1374 interactions (Fig. 7C; Lee et al. 2002; Stark et al. 2006). Searching the metanetwork resulted in the identification of six modules that were significantly enriched over random, shown in Figure 7D (see the Materials and Methods). Modules such as two kinases binding a common protein (module 2) are suggestive of either scaffold protein or coordinate regulation. Others, such as modules 5 and 6 , indicate that proteins bound by the same kinase are often bound by the same transcription factor, suggesting they are subjected to common regulatory mechanisms. A number of kinase-protein interactions from these modules were validated, as summarized in the legend for Figure 7.

\section{Gene Ontology (GO) analysis of protein-kinase interactions}

We also searched for enrichment of GO terms in the sets of all kinase targets (see Supplemental Table S5); the cytoplasm was the only significant GO term in the cellular component GO category. We further analyzed the targets that interacted with the taxonomically related kinases to search for enrichment across biological processes, functions, and cellular components. The CAMK 


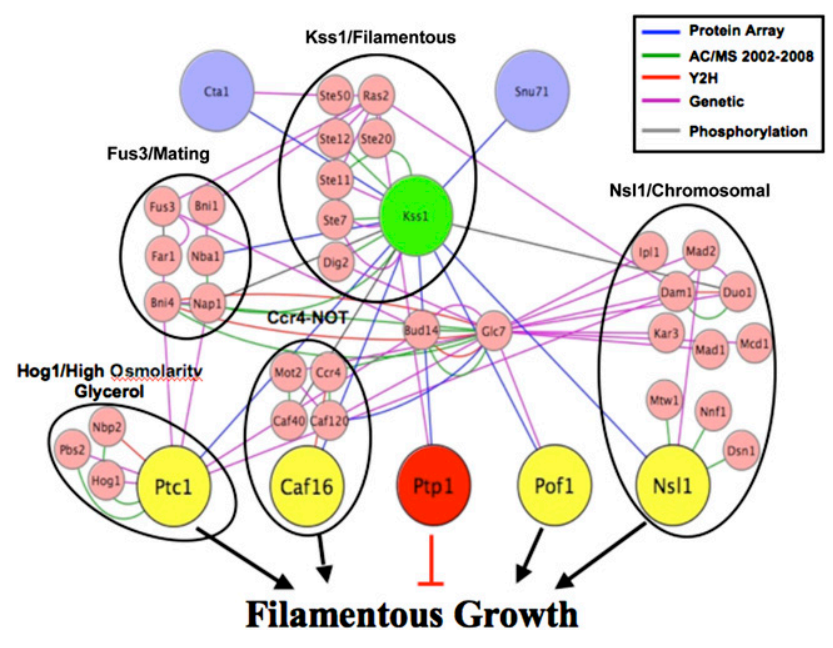

Figure 5. Kss1 targets identified in our study connect to components that interact with the Kss1 filamentous pathway. Large nodes indicate proteins identified on the protein microarray and validated by co-IP/immunoblot to interact with Kss1. Components from the Fus3/Mating, Hog1/osmostress, Ccr4Not, and kinetochore checkpoint interaction profiles have common edges with targets that promote invasive growth. Snu71 does not have any common extended network of interactions that overlap with the proteins in our search space; however, Cta1 does interact genetically with Ras2, which is upstream of the Kss1 pathway. Yellow nodes and the red node (Ptp1) indicate proteins that induced and inhibited filamentous growth, respectively, in the current study (see Fig. 6). Interactions in the extended network were obtained from the Saccharomyces Genome Database (http://www.yeastgenome. org).

group of kinases were found to be enriched for targets involved in a variety of cellular $(P$-value $=2.14 \mathrm{E}-08)$, metabolic $(P$-value $=5.42 \mathrm{E}-07)$, catabolic $(P$-value $=$ $1.54 \mathrm{E}-06)$, and biosynthetic $(P$-value $=2.95 \mathrm{E}-04)$ processes. GO analysis for function resulted in an enrichment in catalytic $(P$-value $=1.78 \mathrm{E}-06)$, transferase $(P$-value $=1.40 \mathrm{E}-04)$, and binding $(P$-value $=8.12 \mathrm{E}-04)$ proteins (see Supplemental Fig. S4; Supplemental Table S6). Thus, the CAMKs interact with metabolic enzymes, consistent with their prominent role in these processes (e.g., Snf1's transcriptional regulation of genes that function in carbon metabolism) (Hardie et al. 1998).

\section{Discussion}

\section{Identification of new interacting partners using protein microarrays}

The information derived from genome- and proteomewide experiments with Saccharomyces cerevisiae have accelerated the rate at which genetic and biochemical information concerning proteins can be integrated into comprehensive network models. Protein microarrays provide a useful, rapid, and unbiased approach to identifying novel protein-protein interactions. In the present study, we performed the largest protein-protein interaction study to date using proteome microarrays and identified
$>1000$ new interactions. Co-IP experiments using protein expressed at endogenous levels validated 33 out of the 45 $(\sim 73 \%)$ novel interactions identified across a range of intensity thresholds. We presume that this is the minimum accuracy, because some of the interactions that did not validate might occur under other growth conditions. This suggests that the majority of interactions that we identified are likely to occur in vivo.

The interactions discovered using protein microarrays are distinct from those identified by other methods (e.g., AC/MS and two-hybrid) (Yu et al. 2008; Braun et al. 2009). Protein microarrays have the advantage that they can detect many low-abundance interactions, which might not be readily identified using AC/MS and two-hybrid experiments. Indeed, many of the interactions we detected involve low-abundance proteins (Ghaemmaghami et al. 2003). Moreover, many of the interactions are likely to be direct, although, since the proteins are purified from yeast, indirect interactions are possible. One disadvantage of protein microarrays is that the interactions occur in vitro and thus might not be present in vivo (although, as noted above, our validation experiments suggest that most interactions occur in vivo). In addition, AC/MS experiments are more likely to find indirect interactions. Overall, these different studies indicate that the various approaches are complementary: Each has a very high false-negative rate (Yu et al. 2008), and thus each approach contributes significant information concerning protein-protein interactions.

\section{Novel connection in the filamentation pathway}

Our analysis of Kss1 and the filamentation pathway revealed a large number of novel interactions. Many of these indirectly involve Glc7, a regulator of glucose metabolism (Cullen and Sprague 2002). Since glucose metabolism and filamentation are linked, such links are consistent with a connection between the Kss1 and Glc7 pathways. Pof1 is a protein that associates physically with Kss1, shares genetic interactions with Glc7 (Costanzo et al. 2010), and is ideally suited to serve as a link connecting these two pathways. Kss1 also interacts with the Ccr4-Not transcriptional/translational regulatory complex and the Nsll subunit of the MIND kinetochore complex. Interestingly, other components of the Ccr4-Not complex have been implicated previously in the filamentous pathway by other studies. For example, the not 1 and not 2 mutants are defective in filamentous growth in S. cerevisiae (Collart 2003), and Candida albicans containing a deletion of NOT4 failed to form hyphae (Krueger et al. 2004). In addition, there are also linkages between Glc7 and the Ccr4-Not pathway (Lenssen et al. 2005).

The interaction between Kss1 and Ns11, a kinetochore component, was unexpected. When yeast undergoes haploid-invasive growth, there is an accompanying G2/M cell cycle delay (Kron et al. 1994), similar to ns11-16 and ns11-42 mutants (Scharfenberger et al. 2003). Perhaps Kss1 functions with kinetochore components to help mediate this delay/checkpoint. These results suggest 
A
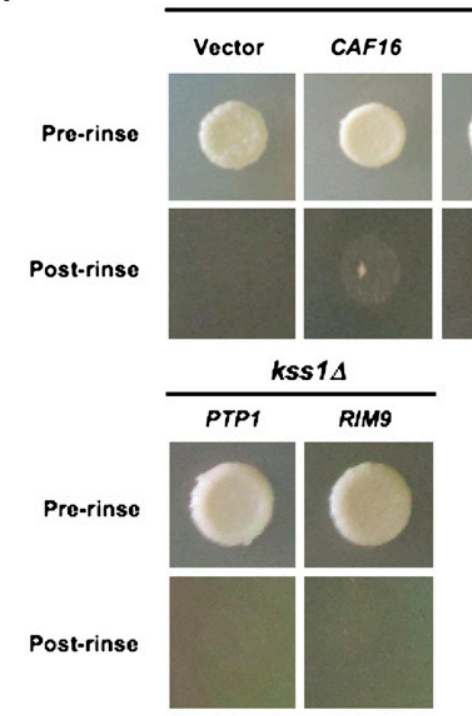

kss14

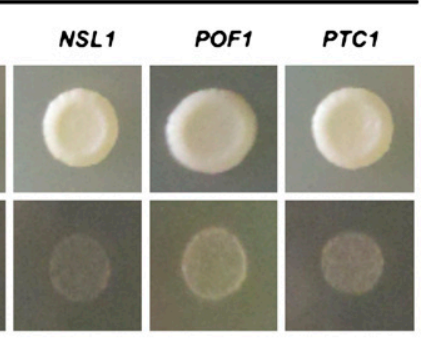

Wild-type

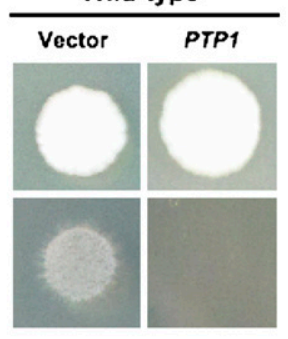

B

Pre-Induction

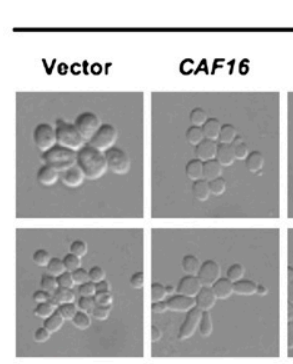

kss1A

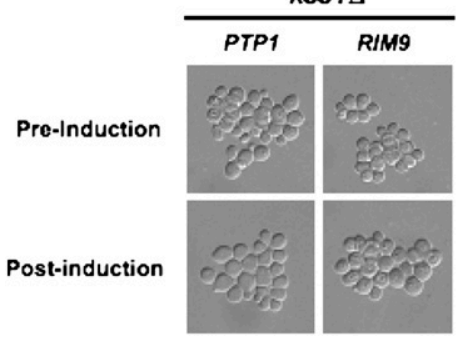

kss14

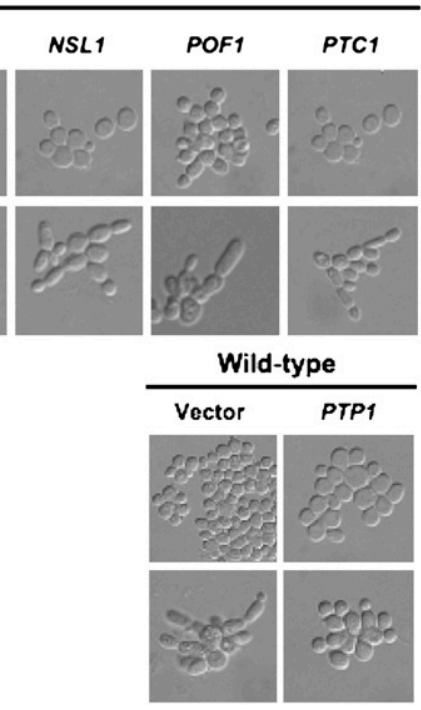

Figure 6. Phenotypic analysis of Kss1 target overexpression in the $\Sigma 1278$ b background reveals new signaling components contributing to haploid filamentous growth. $(A)$ Yeast cells were transformed with $2 \mu$ pYES-DEST5 2 plasmids containing ORFs for the proteins that were validated (at their endogenous levels) by co-IP with Kss1. The effects of overexpressing target proteins on haploid-invasive growth were tested in kss1s and wild-type strains (see the Materials and Methods). The yeast strains labeled vector (empty vector) and RIM9 are negative controls (as were CTA1 and $S N U 71$ ) (data not shown), and KSS1 overexpression serves as a positive control for filamentous growth. CAF16, NSL1, PTC1, and POF1 were sufficient to induce the filamentous pathway in the kss1s cells. The invasive growth phenotype was not observed for overexpression of PTP1 in wild-type cells compared with the negative controls. PTP1 overexpression inhibited the invasive growth phenotype in wild-type cells. (B) DIC images of yeast cells preinduction and post-induction with 3XYEP/ $2 \%$ galactose in liquid culture exhibited the haploid pseudohyphal filamentous phenotype, in agreement with proteins that induce invasive growth. The kss1s yeast cells transformed with pYES-DEST52 high-copy CAF16, KSS1, NSL1, PTC1, and POF1 constructs all formed pseudohyphae compared with the vector control. The cells transformed with similar constructs for PTP1 and RIM9 did not form pseudohyphae. Cells transformed with pYES-DEST52 PTP1 suppressed pseudohyphal growth in the $\Sigma 1278$ b wild-type background. SNU71 and CTA1 overexpression had no effect on the filamentous/haploid-invasive growth phenotype in either the knockout or wild-type strains (data not shown). links with many cellular processes, as might be expected for a key regulatory kinase that controls filamentation. Filamentation is activated upon nutrient stress responses, which would be expected to exert a global effect on cellular activities. The broad interactions of Kss1 with components of different cellular pathways are consistent with this global regulatory response.

Interestingly, our results also suggest cross-regulation of the Kss1 and Hog1 MAP cascades through the activities of Ptc1. A requirement of Hog1 pathway activation is that the Kss1 pathway is rendered inactive and vice versa: Activation of the Kss1 pathway inactivates Hog1 (Shock et al. 2009). Ptcl inactivates the osmosensing MAPK cascade by dephosphorylating Hog1 (Krantz, et al. 2009). Overexpression of PTC1 rescues invasive growth in kss1 cells, indicating that it also functions either downstream from or in parallel to the Kss1 pathway.

\section{Conclusions}

The myriad of interactions contributing to the complexities of eukaryotic cell signaling will require a wide variety of approaches to better understand these networks. Our study to identify a vast repertoire of protein-protein interactions that have not been found previously reveals many connections between cellular pathways. Our results are consistent with a highly connected web of interactions that coordinates cellular activities with intercellular and extracellular responses. Finally, since signaling components are conserved throughout eukaryotes, the results from our study are expected to be applicable to the understanding of signaling networks in other eukaryotes. MAPKs have been proposed to have an evolutionarily conserved role in the cross-regulation of cellular signaling pathways to effect global control in response to external stimuli (Weston 
A.

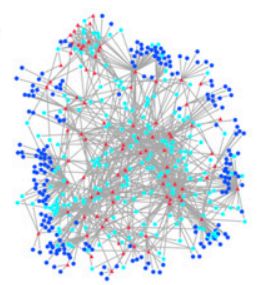

B.

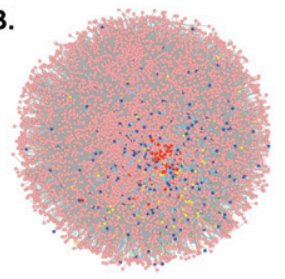

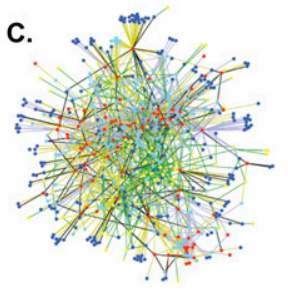

Figure 7. The protein microarray kinase interaction network intersects multiple transcription factor, protein-protein, and phosphorylation data sets to reveal novel network modules. Red nodes are kinases, yellow nodes are transcription factor interactions, dark-blue nodes are single kinase interactions, and light-blue nodes are multiple kinase interactions. (A) Protein microarray global interaction network. There are 21 kinase-kinase interactions and 800 kinase-target-kinase interactions, in which a target is shared by at least two kinases. $(B)$ The union of the kinase array data, transcription factor data $(\mathrm{tf})$, and BioGRID protein interaction data, while keeping only unique interactions, results in a meganetwork incorporating multiple phosphorylation, transcription factor, and BioGRID proteinprotein interaction data sets that intersect the protein microarray network. $(C)$ The metanetwork demonstrating all enriched interactions that intersect our data set and reveal new statistically significant biological modules with the interactions identified in the current study. (D) Summary of overenriched modules identified from analysis of networks (also see Supplemeental Table S4).

et al. 2002; Breitkreutz et al. 2010), and our studies with Kss1 strongly support this concept.

\section{Materials and methods}

\section{Kinase-V5 probe preparation}

Full-length kinase ORFs were cloned into the pYES-DEST52 gateway vector (Invitrogen) from constructs either obtained from the MORF collection or cloned de novo as described previously by Gelperin et al. (2005). The pYES-DEST52 plasmid contains an LR cloning site flanked by a V5-His6X C-terminal tag under control of a GAL1 promoter. The resulting kinase-V5-His6X fusion protein has a His6X tag for protein purification and a 14amino-acid V5 epitope tag (GKPIPNPLLGLDST) for antibody detection. Sequence-verified plasmids were transformed into yeast (Y258: MATa, pep4-3, his4-580, ura3-52, leu2-3, 112) using a standard lithium acetate transformation protocol as described previously (Kumar et al. 2000), and the resulting strains were used to purify the V5-kinase fusion proteins (see Supplemental Table S7 for strains used in this study). Yeast cultures were grown overnight at $30^{\circ} \mathrm{C}$ in $6 \mathrm{~mL}$ of $\mathrm{Sc}-\mathrm{Ura} / 2 \%$ dextrose and diluted to OD600 $=0.1$ the next day in $400 \mathrm{~mL}$ of Sc-Ura/2\% raffinose. Cultures were grown at $30^{\circ} \mathrm{C}$ to $\mathrm{OD} 600 \approx 0.6$ and induced with 3 XYEP $(3 \%$ yeast extract, $6 \%$ peptone, $6 \%$ galac- tose) for $6 \mathrm{~h}$, followed by centrifugation, washing with ice-cold water, and freezing overnight at $-80^{\circ} \mathrm{C}$. Crude lysates were prepared by lysing cells twice with $350 \mathrm{~mL}$ of lysis buffer $(1 \times$ PBS at $\mathrm{pH} 7.4,150 \mathrm{mM} \mathrm{NaCl}, 2 \mathrm{mM} \mathrm{MgCl}, 0.5 \mathrm{mM}$ DTT, $0.1 \%$ Triton X-100, 0.3\% sarkosyl, 0.5\% deoxycholate, $1 \mathrm{mM}$ PMSF, $1 \times$ complete EDTA-free protease inhibitors [Roche], 1:100 dilution phosphatase inhibitor cocktail 1 [Sigma], $100 \mu \mathrm{M}$ sodium orthovanadate) by shaking for $6 \mathrm{~min}$ in a paint shaker (5G-HD, Harbil) at $4^{\circ} \mathrm{C}$ with $250 \mu \mathrm{L}$ of $0.5-\mathrm{mm}$ glass beads (BioSpec Products), followed by centrifugation at $2500 \mathrm{~g}$ for $5 \mathrm{~min}$. Between $2 \mathrm{~g}$ and $10 \mathrm{~g}$ of cell pellets was used to normalize kinasespecific protein yield (microgram per milliliter) from extraction. The lysate was then centrifuged at $150,000 \mathrm{~g}$ for $1 \mathrm{~h}$ at $4^{\circ} \mathrm{C}$ and passed through a nickel $\left(\mathrm{Ni}^{2+}\right)$ sepharose column (His Spintrap, GE Life Sciences) to bind the kinase-V5-His6X fusion protein. The column was washed three times with wash buffer $(1 \times$ PBS at pH 7.4, $500 \mathrm{mM} \mathrm{NaCl}, 2 \mathrm{mM} \mathrm{MgCl} 2,0.1 \%$ Triton X-100, $50 \mathrm{mM}$ imidazole at $\mathrm{pH} 7.4)$, and the protein was eluted with elution buffer $(1 \times$ PBS at $\mathrm{pH} 7.4,500 \mathrm{mM} \mathrm{NaCl}, 2 \mathrm{mM} \mathrm{MgCl} 2,0.1 \%$ Triton X-100, 0.5 mM DTT, 500 mM imidazole, $20 \%$ glycerol).

\section{Protein microarray screen}

Commercial yeast protein microarrays (Invitrogen) containing $\sim 4200$ full-length GST fusion proteins spotted in duplicate were 
used in this screen (Zhu et al. 2001). Arrays were blocked for $1 \mathrm{~h}$ at $4^{\circ} \mathrm{C}$ with shaking in blocking buffer $(1 \times \mathrm{PBS}$ at $\mathrm{pH} 7.4,1 \%$ BSA, $0.1 \%$ Tween-20). Purified kinase-V5 probe was then diluted in probing buffer $(1 \times \mathrm{PBS}, 5 \mathrm{mM} \mathrm{MgCl} 2,0.5 \mathrm{mM} \mathrm{DTT}, 0.05 \%$ Triton X-100, 1\% glycerol, 1\% BSA) augmented with $150 \mathrm{mM}$ $\mathrm{NaCl}$ and $200 \mu \mathrm{M}$ ATP. After blocking, $90 \mu \mathrm{L}$ of the dilute kinase-V5 was loaded onto the microarray membrane at a final concentration of $\sim 10-50 \mu \mathrm{g} / \mathrm{mL}$ with a pipette tip, and the membrane was covered using a Lifterslip (Electron Microscopy Sciences) during probing. The arrays were then incubated in a humidified chamber for $1.5 \mathrm{~h}$ static (no shaking) at $4^{\circ} \mathrm{C}$, followed by washing three times for $1 \mathrm{~min}$ (static) in $25 \mathrm{~mL}$ of probe buffer on ice. The arrays were then probed with $90 \mu \mathrm{L}$ of anti-V5-AlexaFluor647 antibody (Invitrogen) at a concentration of $260 \mathrm{ng} / \mathrm{mL}$ diluted in probing buffer, covered with a Lifterslip as before, and placed back into the humidified chamber for 30 min static at $4{ }^{\circ} \mathrm{C}$. Following antibody detection, the arrays were washed in $25 \mathrm{~mL}$ of probe buffer as before and spun at $800 \mathrm{~g}$ for $5 \mathrm{~min}$ in a tabletop centrifuge to dry. The protein-protein interactions were detected using a 4200AL microarray scanner (Axon Instruments). The image file was then processed using Genepix software (Axon Instruments) to determine signal intensity of the bound kinase probe detected by the anti-V5 AlexaFluor647-conjugated antibody.

\section{Data analysis}

An algorithm called ProCAT (Zhu et al. 2006) was used to identify proteins bound to the kinase probe. Briefly, the program normalizes the background by considering a local window to account for spatial and other artifacts. After a set of filtering steps, the signal of each spot is computed by accounting for the background and foreground signal of the neighboring spots in a three-by-three-feature window. To detect positive spots, the normalized signal is then compared with the neighboring spots in a nine-by-nine-feature window. The number of normalized standard deviations (SDs) above the median signal in the neighboring spots was used to set the threshold to detect positive signals above background. ProCAT has been shown to improve the signal-to-noise ratio of functional protein microarrays (Zhu et al. 2006). We then implemented a two-step learning algorithm to determine the optimal thresholds for the high-quality kinase interactions. For the training set, we used all known kinase interactions in the literature from the BioGRID database as goldstandard positives, and protein pairs with different subcellular localizations as the gold-standard negatives. We employed the naïve Bayesian method as the learning kernel. At the first step, we determined the optimal normalized SD value of intervals for high-quality kinase interactions. Then, in the second step, we determined the specific interactions among the high-quality ones from the first step. The optimal conditions were determined to be a signal-to-noise interval that corresponded to targets validated by co-IP/immunoblot corresponding to ProCAT normalized SD values $(5 \leq \mathrm{SD} \leq 20)$ ]. Interactions bound by this interval were filtered by the number of kinases that interact with the targets ( $\leq 10$ kinases) and subcellular localization. The overlapping targets between kinases form the hubs of interconnectivity, connecting the edges of each kinase to its corresponding targets.

\section{Interaction validation by co-IP}

Co-IP strains were generated by introducing a 13XMyc genomic tag onto the $\mathrm{C}$ terminus of each kinase in the BY4741 (MATa

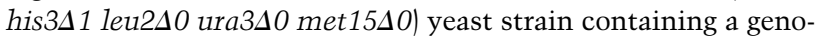
mic TAP tag (Open Biosystems) for each of the interacting proteins identified in the screen (Supplemental Table S7). Additionally, negative control strains were generated for all kinases tested that contained the genomically tagged 13XMyc kinase without TAP-tagged proteins to control for cross-reactivity of the anti-Myc antibody. A genomic $13 \mathrm{XMyc}$ tag was generated using pFA6a-13Myc-KanMX6, and the reverse primer 5' -(gene-specific sequence) GAATTCGAGCTCGTTTAAAC-3' and forward primer 5 '-(gene-specific sequence) CGGATCCCCGGGTTAAT TAA-3' (Longtine et al. 1998) were generated using genomic BY4741 gDNA as a template and Pfx platinum polymerase (Invitrogen).

The co-IP strains were used to inoculate 10-mL YPAD starter cultures overnight, followed by inoculating 500-mL YPAD cultures to $\mathrm{OD} 600=0.1$. Cultures were grown for $7 \mathrm{~h}$ at $30^{\circ} \mathrm{C}$, harvested by centrifugation, washed with cold water, and frozen at $-80^{\circ} \mathrm{C}$. The next day, $2 \mathrm{~g}$ of cell pellet for both the control strain (TAP tag) and the experimental strain (co-IP strain) was parsed over four separate lysis tubes containing $0.5 \mathrm{~g}$ of pellet $(2 \mathrm{~g}$ total), and each was used to prepare the lysates for co-IP.

Lysates were obtained by lysing each of the 0.5-g cell pellets twice in $400 \mathrm{~mL}$ of RIPA buffer $(0.5 \mathrm{M}$ Tris- $\mathrm{HCl}$ at $\mathrm{pH} 7.4,1.5 \mathrm{M}$ $\mathrm{NaCl}, 2.5 \%$ deoxycholic acid, 10\% NP-40, $10 \mathrm{mM}$ EDTA, $1 \mathrm{mM}$ PMSF, $1 \times$ complete protease inhibitors [Roche], 1:100 dilution phosphatase inhibitor cocktail 1 [Sigma], $100 \mu \mathrm{M}$ sodium orthovanadate) by shaking for $1 \mathrm{~min}$, followed by $2 \mathrm{~min}$ on ice in a Fastprep (MP Biomedicals) at $4^{\circ} \mathrm{C}$ with $250 \mu \mathrm{L}$ of glass beads (0.5 mm; BioSpec Products) for a total of three intervals. The homogenate was centrifuged at 14,000 rpm using a microfuge, and the supernatant for each sample was collected. This process was repeated for a total of two extractions, and the lysates from 2 $\mathrm{g}$ of cell pellets were combined in a $15-\mathrm{mL}$ conical tube for co-IP. The co-IPs were performed by incubating $25 \mu \mathrm{L}$ of dynabeads (Invitrogen) conjugated with rabbit IgG (Sigma) with the lysates for $1.5 \mathrm{~h}$ on a nutator at $4^{\circ} \mathrm{C}$. The dynabeads were then washed five times for $10 \mathrm{~min}$ with ice-cold wash buffer (50 mM Tris-HCl at $\mathrm{pH} 7.4,0.1 \%$ Triton $\mathrm{X}-100,150 \mathrm{mM} \mathrm{NaCl}$ ), and beads were boiled at $95^{\circ} \mathrm{C}$ in $65 \mu \mathrm{L}$ of $2 \times$ SDS sample buffer.

The samples were then run out on 10\% NuPAGE gels (Invitrogen) and transferred to nitrocellulose membrane for immunoblotting. The membranes were incubated with 1:1000 dilution of clone 9E10 anti-Myc antibody (Millipore) in 3\% milk/TBS overnight at $4^{\circ} \mathrm{C}$. The next day, the membranes were washed, followed by incubation for $1 \mathrm{~h}$ at room temperature with 1:10,000 dilution of secondary anti-mouse antibodies conjugated to HRP (GE Biosciences) and exposed to Picowest (Pierce) for detection. Co-IP/immunoblot-validated kinase protein-protein interactions were scanned from multiple experiments to create the panels shown in Figure 2. Those interactions that did not validate by co-IP are shown in Supplemental Table S2.

\section{Kinase phosphorylation and binary protein interactions}

To determine which kinase-binding mechanism gave rise to an interaction found by the protein array, $S$. cerevisiae phosphosite data from the literature (Ficarro et al. 2002; Gruhler et al. 2005; Li et al. 2007; Albuquerque et al. 2008; Beltrao et al. 2009; Stark et al. 2010) were incorporated into the protein array-derived kinase network. Kinase position weight matrices (PWMs) from Mok et al. (2010) were used to score every phosphosite. For each kinase PWM, the best score for each phosphoprotein was used to establish a best score background distribution. We assumed the background distribution follows a Student's $t$-distribution and applied a stringent threshold of $P<10^{-20}$ to annotate an interaction as a kinase-phosphorylating interaction. Interactions that failed the threshold were annotated as kinase-binding interactions. 


\section{Network modules analysis}

The kinase network was augmented with transcription factorbinding data (Lee et al. 2002). BioGRID protein interactions version 3.1.71 was used to attain interactions between kinase and transcription factor protein targets (Stark et al. 2006). The interaction network was mined for network modules by a custom in-house $\mathrm{C}++$ program. The program uses a motif-mapping approach by starting from a set of directed nonisomorphic graphs provided by gtools toolset version 2.4 (McKay 1981). A depthfirst approach was used to find each motif in the network. To establish network module significance, 500 random networks were generated. Each class of network interaction was randomized independently, while maintaining each protein's in degree and out degree. Since the kinase network was comprised of two interaction types, a similar ratio of the two interaction types was maintained in the random networks. The one-sided Wilcoxon signed-rank test was used to determine network module significance.

\section{Phenotypic analysis of Kss1 targets}

The ORFs coding for the proteins confirmed to interact with Kss 1 by co-IP/immunoblot were cloned into the pYES-DEST52 gateway plasmid (Invitrogen) as described previously (Gelperin et al. 2005) and transformed into the yeast strain Y825 (MATa ura3-52 leu2 $\Delta 0$ ) derived from the filamentous $\Sigma 1278 \mathrm{~b}$ background (Jin et al. 2008). The pYES-DEST52 plasmid contained a GAL1-inducible promoter to drive expression of the cloned ORF as a V5-His6X fusion protein. The Y825 cells transformed with pYES-DEST52 plasmids containing the ORFs identified in the screen were grown overnight in Sc-Ura/2\% dextrose and were used to inoculate $5-\mathrm{mL} \mathrm{Sc-Ura} / 2 \%$ raffinose $/ 0.1 \%$ dextrose cultures at OD600 $=0.1$. Cells were grown to a density of OD600 of $\sim 0.4$ for $7 \mathrm{~h}$ at $30^{\circ} \mathrm{C}$, plated on $\mathrm{Sc}-\mathrm{Ura} / 2 \%$ galactose $/ 0.2 \%$ raffinose agar plates, and incubated for $3 \mathrm{~d}$ at $30^{\circ} \mathrm{C}$, followed by plate washing to remove yeast above the agar to reveal invasive growth as described previously (Cook et al. 1996). The yeast strains used in the invasion assay were analyzed by microscopy using DIC optics to observe morphological changes that correspond to haploid-invasive growth. Yeast cells grown overnight in $\mathrm{Sc}-\mathrm{Ura} / 2 \%$ dextrose starter cultures were used to inoculate cultures grown in $5 \mathrm{~mL}$ of $\mathrm{Sc}$-Ura $/ 2 \%$ raffinose $/ 0.1 \%$ dextrose for $8 \mathrm{~h}$ at $30^{\circ} \mathrm{C}$ with shaking until cells reached an OD600 of $\sim 0.6$. The cultures were induced for an additional $7 \mathrm{~h}$ at $30^{\circ} \mathrm{C}$ with shaking with 3 XYEP as before. Samples were fixed in $70 \%$ ethanol before and after the induction period prior to imaging. The cell images were captured for samples obtained before and after induction and compared with vector and negative controls for the haploid pseudohyphal phenotype.

\section{Acknowledgments}

We thank Jennifer Gallagher for discussion advice and comments. We are grateful to Anuj Kumar's laboratory for providing us with $\Sigma 1278$ b yeast strains. The NIH supported this work.

\section{References}

Albuquerque CP, Smolka MB, Payne SH, Bafna V, Eng J, Zhou H. 2008. A multidimensional chromatography technology for in-depth phosphoproteome analysis. Mol Cell Proteomics 7: 1389-1396.

Barral Y, Parra M, Bidlingmaier S, Snyder M. 1999. Nim1-related kinases coordinate cell cycle progression with the organization of the peripheral cytoskeleton in yeast. Genes Dev 13: 176-187.
Beltrao P, Trinidad JC, Fiedler D, Roguev A, Lim WA, Shokat KM, Burlingame AL, Krogan NL. 2009. Evolution of phosphoregulation: comparison of phosphorylation patterns across yeast species. PLoS Biol 7: e1000134. doi: 10.1371/ journal.pbio.1000134.

Braun P, Tasan M, Dreze M, Barrios-Rodiles M, Lemmens I, Yu H, Sahalie J, Murray R, Roncari L, de Smet A, et al. 2009. An experimentally derived confidence score for binary proteinprotein interactions. Nat Methods 6: 91-97.

Breitkreutz A, Choi H, Sharom J, Boucher L, Neduva V, Larsen B, Lin Z, Breitkreutz B, Stark C, Liu G, et al. 2010. A global protein kinase and phosphatase interaction network in yeast. Science 328: 1043-1046.

Collart M. 2003. Global control of gene expression in yeast by the Ccr4-Not complex. Gene 313: 1-16.

Cook J, Bardwell L, Kron S, Thorner J. 1996. Two novel targets of the MAP kinase Kss1 are negative regulators of invasive growth in the yeast Saccharomyces cerevisiae. Genes Dev 10: $2831-2848$.

Cook J, Bardwell L, Thorner J. 1997. Inhibitory and activating functions for MAPK Kss 1 in the $S$. cerevisiae filamentousgrowth signalling pathway. Nature 390: 85-88.

Costanzo M, Baryshnikova A, Bellay J, Kim Y, Spear E, Sevier C, Ding H, Koh J, Toufighi K, Mostafavi S, et al. 2010. The genetic landscape of a cell. Science 327: 425-431.

Costigan C, Snyder M. 1994. SLK1, a yeast homolog of MAP kinase activators, has a RAS/cAMP-independent role in nutrient sensing. Mol Gen Genet 243: 286-296.

Cullen P, Sprague GJ. 2002. The Glc7p-interacting protein Bud14p attenuates polarized growth, pheromone response, and filamentous growth in Saccharomyces cerevisiae. Eukaryot Cell 1: 884-894.

Erdman S, Snyder M. 2001. A filamentous growth response mediated by the yeast mating pathway. Genetics 159: 919-928.

Ficarro SB, McCleland ML, Stukenberg PT, Burke DJ, Ross MM, Shabanowitz J.Hunt DF, White FM. 2002. Phosphoproteome analysis by mass spectrometry and its application to Saccharomyces cerevisiae. Nat Biotechnol 20: 301-305.

Gavin A, Bösche M, Krause R, Grandi P, Marzioch M, Bauer A, Schultz J, Rick J, Michon A, Cruciat C, et al. 2002. Functional organization of the yeast proteome by systematic analysis of protein complexes. Nature 415: 141-147.

Gelperin D, White M, Wilkinson M, Kon Y, Kung L, Wise K, Lopez-Hoyo N, Jiang L, Piccirillo S, Yu H, et al. 2005. Biochemical and genetic analysis of the yeast proteome with a movable ORF collection. Genes Dev 19: 2816-2826.

Ghaemmaghami S, Huh WK, Bower K, Howson RW, Belle A, Dephoure N, O'Shea EK, Weissman JS. 2003. Global analysis of protein expression in yeast. Nature 425: 737-741.

Gruhler A, Olsen JV, Mohammed S, Mortensen P, Faergeman NJ, Mann M.Jensen ON. 2005. Quantitative phosphoproteomics applied to the yeast pheromone signaling pathway. Mol Cell Proteomics 4: 310-327.

Hardie D, Carling D, Carlson M. 1998. The AMP-activated/ SNF1 protein kinase subfamily: metabolic sensors of the eukaryotic cell? Annu Rev Biochem 67: 821-855.

Ho Y, Gruhler A, Heilbut A, Bader G, Moore L, Adams S, Millar A, Taylor P, Bennett K, Boutilier K, et al. 2002. Systematic identification of protein complexes in Saccharomyces cerevisiae by mass spectrometry. Nature 415: 180-183.

Jin R, Dobry C, McCown P, Kumar A. 2008. Large-scale analysis of yeast filamentous growth by systematic gene disruption and overexpression. Mol Biol Cell 19: 284-296.

Kafadar KA, Zhu H, Snyder M, Cyert MS. 2003. Negative regulation of calcineurin signaling by Hrr25p, a yeast homolog of casein kinase I. Genes Dev 17: 2698-2708. 
Kornev A, Taylor S. 2010. Defining the conserved internal architecture of a protein kinase. Biochim Biophys Acta 1804: 440-444.

Krantz M, Ahmadpour D, Ottosson L, Warringer J, Waltermann C, Nordlander B, Klipp E, Blomberg A, Hohmann S, Kitano H. 2009. Robustness and fragility in the yeast high osmolarity glycerol (HOG) signal-transduction pathway. Mol Syst Biol 5: 281. doi: 10.1038/msb.2009.36.

Krogan N, Cagney G, Yu H, Zhong G, Guo X, Ignatchenko A, Li J, Pu S, Datta N, Tikuisis A, et al. 2006. Global landscape of protein complexes in the yeast Saccharomyces cerevisiae. Nature 440: 637-643.

Kron S, Styles C, Fink G. 1994. Symmetric cell division in pseudohyphae of the yeast Saccharomyces cerevisiae. Mol Biol Cell 5: 1003-1022.

Krueger K, Ghosh A, Krom B, Cihlar R. 2004. Deletion of the NOT4 gene impairs hyphal development and pathogenicity in Candida albicans. Microbiology 150: 229-240.

Kumar A, des Etages SA, Coelho PS, Roeder GS, Snyder M. 2000. High-throughput methods for the large-scale analysis of gene function by transposon tagging. Methods Enzymol 328: 550 574.

La Valle R, Wittenberg C. 2001. A role for the Swe1 checkpoint kinase during filamentous growth of Saccharomyces cerevisiae. Genetics 158: 549-562.

Lee TI, Rinaldi NJ, Robert F, Odom DT, Bar-Joseph Z, Gerber GK, Hannett NM, Harbison CT, Thompson CM, Simon I, et al. 2002. Transcriptional regulatory networks in Saccharomyces cerevisiae. Science 298: 799-804.

Lenssen E, James N, Pedruzzi I, Dubouloz F, Cameroni E, Bisig R, Maillet L, Werner M, Roosen J, Petrovic K, et al. 2005. The Ccr4-Not complex independently controls both Msn2dependent transcriptional activation-via a newly identified Glc7/Bud14 type I protein phosphatase module-and TFIID promoter distribution. Mol Cell Biol 25: 488-498.

Li X, Gerber SA, Rudner AD, Beausoleil SA, Haas W, Villen J, Elias JE, Gygi SP. 2007. Large-scale phosphorylation analysis of $\alpha$-factor-arrested Saccharomyces cerevisiae. I Proteome Res 6: 1190-1197.

Longtine MS, McKenzie A III, Demarini DJ, Shah NG, Wach A, Brachat A, Philippsen P, Pringle JR. 1998. Additional modules for versatile and economical PCR-based gene deletion and modification in Saccharomyces cerevisiae. Yeast 14: 953-961.

Lorenz M, Cutler N, Heitman J. 2000. Characterization of alcohol-induced filamentous growth in Saccharomyces cerevisiae. Mol Biol Cell 11: 183-199.

McKay BD. 1981. Practical graph isomorphism. Congressus Numerantium 30: 45-87.

Mok J, Kim PM, Lam HY, Piccirillo S, Zhou X, Jeschke GR, Sheridan DL, Parker SA, Desai V, Jwa M, et al. 2010. Deciphering protein kinase specificity through large-scale analysis of yeast phosphorylation site motifs. Sci Signal 109: ra12. doi: 10.1126/scisignal.2000482.

Popescu S, Popescu GV, Bachan S, Zhang Z, Seay M, Gerstein M, Snyder M, Dinesh-Kumar SP. 2007. Differential binding of calmodulin-related proteins to their targets revealed through high-density Arabidopsis protein microarrays. Proc Natl Acad Sci 104: 4730-4735.

Ptacek J, Devgan G, Michaud G, Zhu H, Zhu X, Fasolo J, Guo H, Jona G, Breitkreutz A, Sopko R, et al. 2005. Global analysis of protein phosphorylation in yeast. Nature 438: 679-684.

Rigaut G, Shevchenko A, Rutz B, Wilm M, Mann M, Séraphin B. 1999. A generic protein purification method for protein complex characterization and proteome exploration. Nat Biotechnol 10: 1030-1032.
Scharfenberger M, Ortiz J, Grau N, Janke C, Schiebel E, Lechner J. 2003. Nsllp is essential for the establishment of bipolarity and the localization of the Dam-Duo complex. EMBO I 22: 6584-6597.

Shock T, Thompson J, Yates JR III, Madhani H. 2009. Hog1 mitogen-activated protein kinase (MAPK) interrupts signal transduction between the Kss1 MAPK and the Tec1 transcription factor to maintain pathway specificity. Eukaryot Cell 8: 606-616.

Stark C, Breitkreutz BJ, Reguly T, Boucher L, Breitkreutz A, Tyers M. 2006. BioGRID: a general repository for interaction datasets. Nucleic Acids Res 34: D535-D539. doi: 10.1093/ nar/gkj109.

Stark C, Su TC, Breitkreutz A, Lourenco P, Dahabieh M, Breitkreutz BJ, Tyers M, Sadowski I. 2010. PhosphoGRID: a database of experimentally verified in vivo protein phosphorylation sites from the budding yeast Saccharomyces cerevisiae. Database (Oxford) 2010: bap026. doi: 10.1093/ database/bap086.

Uetz P, Giot L, Cagney G, Mansfield T, Judson R, Knight J, Lockshon D, Narayan V, Srinivasan M, Pochart P, et al. 2000. A comprehensive analysis of protein-protein interactions in Saccharomyces cerevisiae. Nature 403: 623-627.

van Dongen S. 2000. Graph clustering by flow simulation. $\mathrm{PhD}$ thesis, Utrecht University, Amsterdam, Netherlands.

Vanrobays E, Gleizes P, Bousquet-Antonelli C, Noaillac-Depeyre J, Caizergues-Ferrer M, Gélugne J. 2001. Processing of $20 \mathrm{~S}$ pre-rRNA to $18 \mathrm{~S}$ ribosomal RNA in yeast requires Rrp10p, an essential non-ribosomal cytoplasmic protein. $E M B O J \mathbf{2 0 :}$ 4204-4213.

Weston C, Lambright D, Davis R. 2002. Signal transduction. MAP kinase signaling specificity. Science 296: 2345-2347.

Yu H, Braun P, Yildirim M, Lemmens I, Venkatesan K, Sahalie J, Hirozane-Kishikawa T, Gebreab F, Li N, Simonis N, et al. 2008. High-quality binary protein interaction map of the yeast interactome network. Science 322: 104-110.

Zhu H, Bilgin M, Bangham R, Hall D, Casamayor A, Bertone P, Lan N, Jansen R, Bidlingmaier S, Houfek T, et al. 2001. Global analysis of protein activities using proteome chips. Science 293: 2101-2105.

Zhu X, Gerstein M, Snyder M. 2006. ProCAT: a data analysis approach for protein microarrays. Genome Biol 7: R110. doi: 10.1186/gb-2006-7-11-r110. 


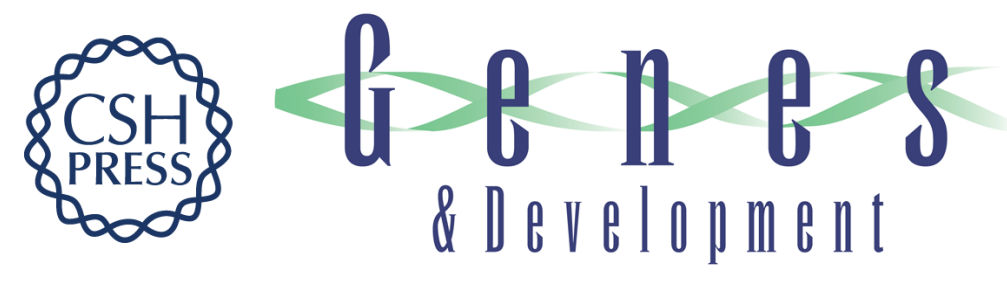

\section{Diverse protein kinase interactions identified by protein microarrays reveal novel connections between cellular processes}

Joseph Fasolo, Andrea Sboner, Mark G.F. Sun, et al.

Genes Dev. 2011, 25:

Access the most recent version at doi:10.1101/gad.1998811

Supplemental
Material $\quad$ http://genesdev.cshlp.org/content/suppl/2011/03/30/25.7.767.DC1

References This article cites 48 articles, 22 of which can be accessed free at: http://genesdev.cshlp.org/content/25/7/767.full.html\#ref-list-1

License Freely available online through the Genes \& Development Open Access option.

Email Alerting Receive free email alerts when new articles cite this article - sign up in the box at the top Service right corner of the article or click here.

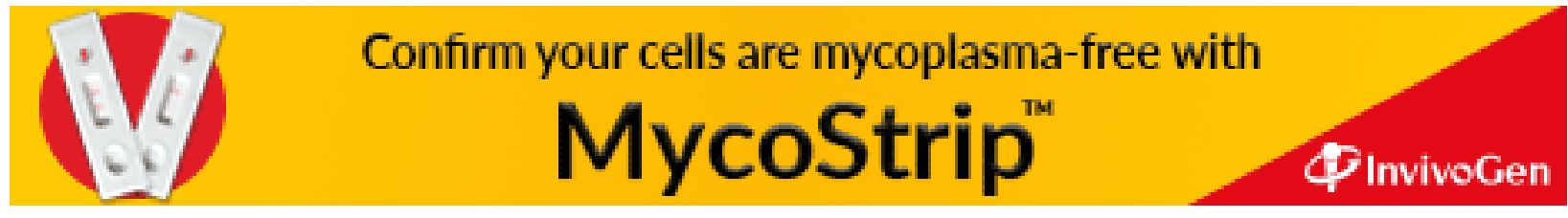

